\title{
Mathematical modeling of respiratory system mechanics in the newborn lamb
}

\author{
Virginie Le Rolle • Nathalie Samson • \\ Jean-Paul Praud • Alfredo I. Hernández
}

Received: date / Accepted: date

\begin{abstract}
In this paper, a mathematical model of the respiratory mechanics is used to reproduce experimental signal waveforms acquired from three newborn lambs. As the main challenge is to determine specific lamb parameters, a sensitivity analysis has been realized to find the most influent parameters, which are identified using an evolutionary algorithm. Results show a close match between experimental and simulated pressure and flow waveforms obtained during spontaneous ventilation and pleural pressure variations acquired during the application of positive pressure, since Root Mean Square Errors (RMSE) equal to $0.0119,0.0052$ and 0.0094 . The identified parameters were discussed in light of previous knowledge of respiratory mechanics in the newborn.
\end{abstract}

Keywords Model · Animal Experimentation · Sensitivity Analysis . Parameter identification

\section{Introduction}

Due to the complexity of the cardio-respiratory system and its regulation, the analysis of normal respiratory mechanics, as well as diagnosis and treat-

\author{
Virginie Le Rolle \\ INSERM, U1099, Rennes, F-35000, France \\ Université de Rennes 1, LTSI, Rennes, F-35000, France \\ Tel.: +33223235903 \\ Fax: +33223236917 \\ E-mail: virginie.lerolle@univ-rennes1.fr \\ Nathalie Samson \\ Department of Pediatrics, University of Sherbrooke, J1H5N4, QC-Canada \\ Jean-Paul Praud \\ Department of Pediatrics, University of Sherbrooke, J1H5N4, QC-Canada \\ Alfredo I. Hernández \\ INSERM, U1099, Rennes, F-35000, France \\ Université de Rennes 1, LTSI, Rennes, F-35000, France
}


ment of pulmonary diseases may be improved by using mathematical models of the respiratory system [31] [38]. Although several models of the respiratory mechanics have been proposed in the literature over the past 60 years, most of them have been designed to represent adult physiology. One-compartment models can be as simple as the linear, mono-alveolar model [8] [9] or non-linear to differentiate the inspiratory and expiratory phases [6]. Two-compartment models were proposed by Otis et al [29] to account for differences in ventilation between various regions of the lung. Various modifications of the models came from i) identification of the visco-elastic properties of the lung [28] ; ii) serial arrangement of two compartments, i.e. the intermediate airways and the alveoli [24] ; iii) integration of non-linear effects, to account for dynamic airway compression and non-linear behavior of airway resistances [19] [42] ; iv) integration of chest wall mechanics and pleural pressure [2] [3] [21] [22]. Finally, more recent multi-compartment models have been aimed to account for the heterogeneous distribution of ventilation within the lung [43]. Some of these are based on the Weibel representation of the tracheobronchial tree in 24 zones [33] [36].

Respiratory system mechanics of the newborn mammal are very different from the adult and characterized, among others, by a higher lung resistance, a lower lung compliance and a much higher chest wall compliance [11] [27]. Several teams have adapted adult models of respiratory system mechanics, especially to test the accuracy of respiratory mechanics measurement techniques, or the effect of various modes of mechanical ventilation in the newborn. For example, various respiratory mechanics models were used to study the measurement accuracy of respiratory impedance in the newborn [37] or to estimate respiratory resistances and compliances in lambs [7] [8] [9] [18]. Furthermore, models were used to assess the relationships between respiratory mechanics and high-frequency oscillatory ventilation [31] or the bubble CPAP [23]. Finally, some newborn models have been proposed, focused on a single component of the respiratory system, such as the trachea [5] [38].

Although previous studies show the interest of using lumped-parameter models to analyze respiratory system dynamics in mammals, their ability to reproduce real respiratory signal waveforms has not been thoroughly tested. Esophageal pressure and nasal flow has been chosen in this paper since these signals characterize respiratory mechanics. Experimental signals have been acquired on three newborn lambs and the main challenge is to propose a model, which parameters are specific for each lamb. This step requires the identification of specific lamb parameters, that will allow the simulation of respiratory signals approaching as best as possible the experimental data. In order to study respiratory signal profiles, an appropriate description of the respiratory muscle activity must be included in the model, since the dynamics of these muscles determine the respiratory profiles. Reproducing individual respiratory signal waveforms could be useful to assess cardio-pulmonary interactions in our future studies. In fact, the influence of respiratory signal waveforms can be directly observed on cardiovascular signals (arterial pressure, heart rate,...) and the simulation of realistic waveforms will help us to analyze the hemody- 
namic consequences of respiratory devices $(\mathrm{CPAP}, \ldots)$. We previously presented preliminary results on the capacity of a lumped-parameter respiratory model to reproduce real signal waveforms measured in a single newborn lamb [20]. Based on that proof-of-concept account, the aim of the present study is to propose a mathematical model of respiratory system mechanics, adapted to each newborn lamb, which can simulate realistic respiratory signal profiles and reproduce experimental data recorded both during spontaneous, stationary breathing conditions and during transient application of various levels of positive pressure to the airways.

\section{Methods}

2.1 Respiratory model adapted to the newborn

Figure 1 depicts the electrical analog of the proposed non-linear, lumpedparameter model of respiratory mechanics. The global structure is based on a generic two-compartment model, which includes chest wall dynamics [2] [3] [21] [22]. This two-compartment resolution was chosen since it integrates a description of the interaction between upper airways, the lung and chest wall dynamics, while requiring low computational resources. The model consists of the upper airways, the intermediate airways, the lower airways, the alveolar compartment, the pleural cavity and the chest wall.

The upper airways are mainly composed of the nasal passages, the pharynx, the larynx and part of the trachea. In this complex structure, airflow behavior is represented by a non-linear Rohrer resistance

$$
R u=K_{1}+K_{2} \cdot \dot{V}
$$

where $\mathrm{Ru}$ is the upper airways resistance, $K_{1}$ is the resistance component for laminar flow, $K_{2}$ is the resistance component that describes turbulence and $\dot{V}$ is the flow [3] [15] [31]. The smaller inner diameters of newborn upper airways, combined with higher respiratory rates, provoke significant differences in newborn airflow behavior with respect to adults. As a consequence, contrarily to adult models, gas inertia cannot be neglected [15]. In the proposed neonatal model, upper airway pressure $\mathrm{Pu}$ is thus computed as

$$
P u=R u \cdot \dot{V}+I u \cdot \ddot{V}
$$

where Iu the upper airway inertance and $\ddot{\mathrm{V}}$ the acceleration.

The intermediate airways are modeled by a non-linear resistance and a pressure-volume relationship to take into account the volume changes during the respiratory cycle. The resistance varies inversely with the square of airway volume as $R_{c}=K_{3}\left(V_{c \max } / V_{c}\right)^{2}$, where $K_{3}$ is the resistance value when intermediate airway volume $V_{c}$ is equal to its maximum $V_{c \max }$ [14]. The pressurevolume relationship has been determined from experimental data as $V_{c}=$ 


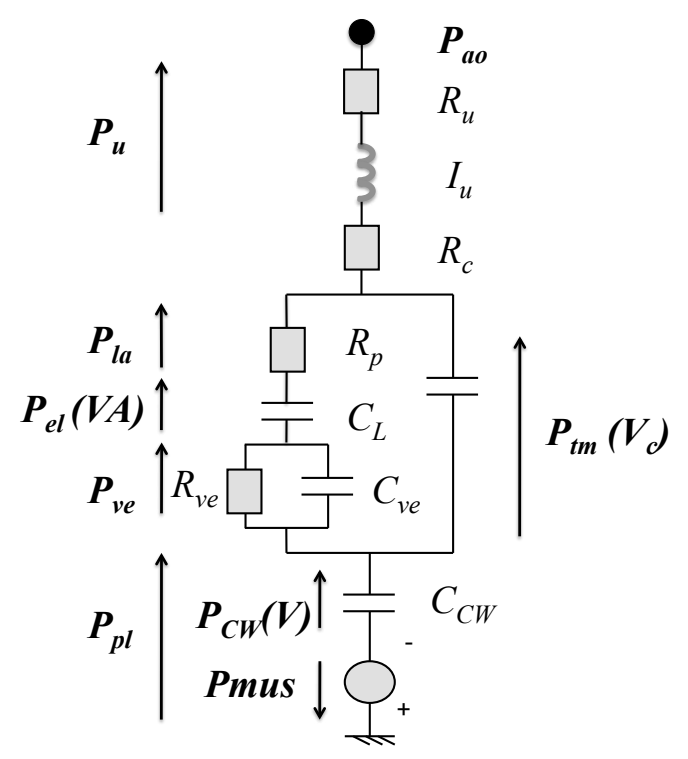

Fig. 1 Electrical analog of the proposed neonatal respiratory system model. The upper airways are characterized by a resistance $R_{u}$ and an inertance $I_{u}$, across which the upper airway pressure $P_{u}$ drops. The intermediate airways are modeled by a resistance $\left(R_{c}\right)$ and compliant relationship between the airways volume $V_{c}$ and the transmural pressure $P_{t m}$. $P_{m u s}$ is the respiratory muscle pressure. $V_{A}$ is the alveolar compartment volume. $P_{l a}, P_{e l}$, $P_{v e}$, and $P_{p l}$ are respectively the lower airways, the lung elastic recoil, the visco-elastic and the pleural pressures. The arrows indicate the pressure directions and the element associated with each pressure.

$V_{\text {cmax }} /\left(1+\exp \left(-\mathrm{a}\left(P_{t m}-\mathrm{b}\right)\right)\right)$, where a and $\mathrm{b}$ are constants and $P_{t m}$ is the transmural pressure, i.e. the difference between intraluminal pressure of the intermediate airways and pleural pressure [3]. While this equation has been determined from adult data, similar curves, describing pulmonary pressure-volume relationships, have shown to be useful in the study of liquid ventilation in newborn lambs [35].

The lower airways are traditionally characterized by laminar airflow, such that a linear resistance $R_{p}$ defines a relation between alveolar flow $\dot{V}_{A}$ and intraluminal pressure $P_{l a}$ :

$$
P_{l a}=R_{p} \cdot \dot{V}_{A}
$$

All alveoli are traditionally modeled by one compartment, which has linear compliant properties such as $P_{e l}=V_{A} / C_{L}$, where $V_{A}$ is the alveolar volume, $C_{L}$ is the lung compliance and $P_{e l}$ is the lung elastic recoil pressure. In order to increase the model applicability in neonatal conditions, the viscoelastic behavior of lung tissue has been taken into account by combining $C_{L}$ in series with a linear compliance $C_{v e}$ and a resistance $R_{v e}$. The alveolar pressure is computed as the sum of lung elastic recoil pressure $P_{e l}$, the visco-elastic pressure $P_{v e}$ 
and pleural pressure $P_{p l}: P_{A}=P_{e l}+P_{v e}+P_{p l}$. The airways and the alveoli are enclosed by a compliant chest wall, whose properties are assumed to be linear, such as $P_{C W}=\mathrm{V} / C_{C W}$, where $P_{C W}$ is the chest wall pressure, $C_{C W}$ is the chest wall compliance and $\mathrm{V}$ is the total respiratory system volume. The pleural pressure is driven by a set of respiratory muscles (Pmus), such as

$$
P_{p l}=P_{C W}-\text { Pmus. }
$$

Previous studies have proposed simplified mechanical models of the respiratory system, including the rib cage and the abdominal compartments [4] and mathematical models of the central respiratory oscillator [10] [39] [40] to represent respiratory muscle activity. Such a description is very useful to study the effects of respiratory rate variations, but the oscillator parameters do not explicitly describe the respiratory signal morphology and thus, reproduction of real respiratory waveforms cannot be performed with this approach. To our knowledge, only a limited number of works have been focused on the modeling of realistic respiratory pressure and flow profiles. In [44] and [34], the influence of the relations between neural output and muscle mechanical performance on the generation of pressure and flow profiles is analyzed. This relation is mainly characterized by seven parameters defining the duration, peak instant and the curvature of the neural output waveform, which are different during the inspiratory and expiratory phases. In order to reproduce different respiratory waveforms, these studies show that it is necessary to take into account parameters describing the neural output morphology that allows for the development of respiratory muscles pressure. In this work, we propose to model directly a realistic Pmus waveform by means of an analytic relation presenting similar characteristics:

$$
\text { Pmus }=P_{\max }\left(B_{0}+\left(1-B_{0}\right) \cdot\left(t_{r} / T_{I}\right)^{\beta} \cdot e^{-\left(\left(t_{r} / T_{I}\right)^{\alpha}+1\right)}\right)
$$

where $P_{\max }$ corresponds to maximum muscle activity, $B_{0}$ is the basal level at end-expiration, $t_{r}$ is the time elapsed from the onset of the current respiratory cycle and $T_{I}$ is the inspiration duration. In addition, parameters $\alpha$ and $\beta$ characterize the Pmus signal profile during inspiration and expiration, respectively. Although the individual contribution of each type of respiratory muscle involved in the generation of the total Pmus are not represented, the proposed analytic function has been designed to fit the different Pmus morphologies generated by the complex and heterogeneous activation of all respiratory muscles. The main advantages of such an analytical representation are related to the fact that different respiratory signal waveforms can be generated by modulating these six parameters, using a single equation. A similar analytic description has been previously applied to the simulation of the ventricular elastance to simulate pulsatile ventricular and arterial pressures [41]. This model has been implemented under a multi-formalism modeling and simulation framework (M2SL), developed in our laboratory, which eases the steps of sensitivity analysis, parameter identification and the integration of the proposed model with other components of the cardiovascular system [16][17]. 
2.2 In vivo experiments

The in vivo experiments were performed in three full-term newborn lambs between three and five days of life. Lamb weights were $3 \mathrm{~kg}, 3.6 \mathrm{~kg}$ and $4.5 \mathrm{~kg}$ respectively. The protocol was approved by the Ethics Committee for Animal Care and Experimentation of the Université de Sherbrooke, Canada. Aseptic surgery was performed under general anesthesia (2\% isoflurane, 30\% N2O, $68 \%$ O2) upon the day of arrival. Anesthesia was preceded by an intramuscular injection of ketamine $\left(10 \mathrm{mg} . \mathrm{kg}^{-1}\right)$, atropine sulfate $\left(0.1 \mathrm{mg} . \mathrm{kg}^{-1}\right)$ and morphine $\left(0,016 \mathrm{mg} \cdot \mathrm{kg}^{-1}\right)$, and an intra-venous bolus $\left(10 \mathrm{mg} \cdot \mathrm{kg}^{-1}\right)$ of Ringer's lactate solution. One dose of ketoprofen $\left(3 \mathrm{mg} \cdot \mathrm{kg}^{-1}\right)$ was also injected intramuscularly for analgesia and repeated if needed after $12 \mathrm{~h}$. Antibiotics $\left(5 \mathrm{mg} \cdot \mathrm{kg}^{-1}\right.$ gentamicin and $0.05 \mathrm{mg} . \mathrm{kg}-1$ duplocilline) were administered intramuscularly prior to surgery and daily thereafter. A microtip pressure transducer catheter (Millar, 3 Fr, Millar Instruments) was inserted into the esophagus, through a small catheter inserted directly into the esophagus during surgery, just before the recording in order to monitor esophageal pressure variations (Pes). Non-calibrated respiratory inductance plethysmography (Respitrace, Ambulatory Monitoring, Ardsley, NY, USA) was used to semi-quantitatively record lung volume variations. All physiological signals were transmitted by wires. On the experimental day (two to three days after surgery), baseline stationary values of mask pressure (Pmask) and airflow $\left(\dot{V}_{\text {mask }}\right)$ were both measured during quiet ventilation through a nasal mask. The pneumotachograph (21070B + 47304A flow transducer, Hewlett Packard, Palo Alto, CA, USA) was connected to the nasal mask. Thereafter, lambs were intubated with a $4.5 \mathrm{~mm}$ endotracheal tube under upper airway anesthesia (lidocaine 4\%). Central apneas were induced by hyperventilation using a Servo 300 ventilator (Siemens Corporation, New-York, NY). No sedation was used. During central apnea, several levels of positive pressure (from $0 \mathrm{cmH} 2 \mathrm{O}$ to $30 \mathrm{cmH} 2 \mathrm{O}$ ) with progressive transitions, at onset and end, were applied on the airways during 15 seconds (CPP15 maneuvers).

2.3 Fitting the model to experimental data and parameter identification

\subsubsection{Processing raw data}

Esophageal pressure and nasal flow were first recorded under stationary breathing conditions in order to characterize typical waveforms of these signals. The sampling frequency was equal to $1000 \mathrm{~Hz}$. All respiratory cycles were then extracted from a period of 20 seconds selected during an epoch of quiet sleep, considering that the onset and end of inspiration occurred when instantaneous airflow was equal to zero. Quiet sleep was defined based on standard behavioral criteria, REM sleep being easy to recognize from quiet sleep by observation in lambs. 
Once all respiratory cycles were extracted, they were registered by resampling all time scales with respect to the longest respiratory cycle with a linear interpolation for both nasal flow and esophageal pressure signals. The correlations between all registered signals and the longest respiratory cycle were computed in order to verify the similarity existing between each signal waveform. The mean correlation coefficients ( $\mathrm{r}$ ) calculated for all respiratory cycles in each lamb were $0.968,0.938$, and 0.935 for nasal flow and $0.969,0.986$ and 0.964 for esophageal pressure. These values indicate that only very similar breathing cycles were included in the registration, limiting distortion of the useful signal.

In order to maximize signal-to-noise ratio, following registration, a mean respiratory cycle for both nasal flow and esophageal pressure was computed in each lamb in stationary conditions. Since one of the goals of the present study was to evaluate the response of $P_{p l}$ to airway application of several levels of positive pressure, Pes was differentiated in order to obtain $P_{p l}$ variations [1] [12]. Finally, a low-pass filter was applied to the differentiated signal to improve signal-to-noise ratio. A 3rd order Butterworth filter, with a cutoff frequency equal to $30 \mathrm{~Hz}$, was thus applied. The second step of the in vivo experiments consisted in the application of various levels of positive pressure to the respiratory system through the endotracheal tube (from $0 \mathrm{~cm} \mathrm{H}_{2} \mathrm{O}$ to $\left.30 \mathrm{~cm} \mathrm{H}_{2} \mathrm{O}\right)$ to assess esophageal pressure variations. In order to solely observe the response of $P_{e s}$ to $P_{a o}$ (pressure at the airway opening), a moving-average filter was applied on 500 samples of the original signal to obtain the lowfrequency component of the signal. The mean $P_{e s}$ was then differentiated in order to obtain induced pleural pressure variations. Finally, the signal-to-noise ratio was improved by applying a 3rd order low-pass Butterworth filter, with a cutoff frequency equal to $30 \mathrm{~Hz}$.

\subsubsection{Sensitivity analysis}

Few studies in the literature have been dedicated to the estimation of accurate physiological parameter values for mathematical models of respiratory mechanics such as $I_{u}, K_{1}, K_{2}, R_{p}$. This is partly due to the fact that the vast majority of these parameters are difficult to obtain in vivo. Table 1 summarizes some of the previously published data, by presenting the main model equations with their corresponding parameter values. Although most data were obtained in adult humans, some parameter values come from studies in newborn lambs or infants, which were based on elementary models of the respiratory system [8] [9]. In order to determine which parameters should be identified from our newborn lamb data, a sensitivity analysis was performed using the screening method of Morris [26], which consists in generating several random trajectories through the parameter space. Each trajectory is associated with an estimation of the Elementary Effects $\left(E E_{k}\right)$, which is defined, for a parameter $x_{k}$ as:

$$
E E_{k}=\left|\left(y\left(x_{1}, \ldots, x_{k}+\Delta, \ldots, x_{n}\right)-y\left(x_{1}, \ldots, x_{k}, \ldots, x_{n}\right)\right)\right| / \Delta
$$




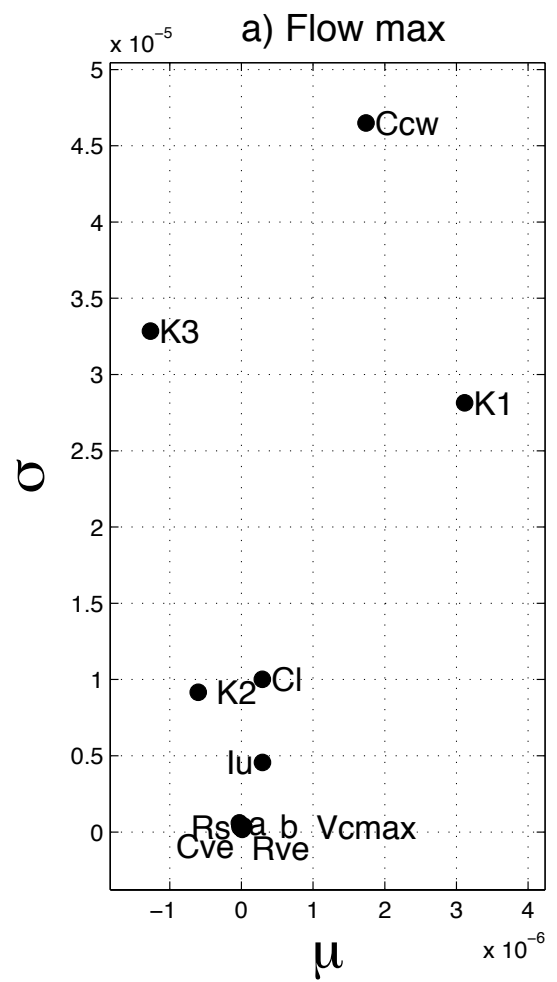

b) $\mathrm{dPpl} / \mathrm{dt} \max$

Fig. 2 Sensitivity analysis results for two model outputs: a) maximum values of flow and b) maximum values of the derivative of pleural pressure during one respiratory cycle. Parameters $\mathrm{a}$ and $\mathrm{b}$ are the constants characterizing the pressure-volume relationship of intermediate airways. $C_{L}, C_{v e}$, and $C_{C W}$ are respectively the lung, the visco-elastic and chest wall compliances. $K_{1}, K_{2}$ and $I_{u}$ are respectively the upper airway resistance coefficient and inertance. $K_{3}, R_{v e}$ and $R_{p}$ are the intermediate, the visco-elastic and the lower airway resistances. $V_{c \max }$ is the maximum value of the intermediate airway volume, which can be computed as the difference between the total volume and the alveolar volume. Vcmax has been approximated by an estimation of the total dead space. The most sensitive parameters are those presenting higher absolute values for $\mu$ and $\sigma$.

where $\Delta=p / 2(p-1), \mathrm{p}$ is defined as the number of levels that divide the parameter space and $\mathrm{y}$ is the model output, which can be each model variable. Two output variables were considered in this analysis: the maximum values of flow and the maximum of the derivative of pleural pressure during one respiratory cycle. The sensitivity analysis provides a mean value $\mu$ and a standard variation $\sigma$ of the set of $E E_{k}$ computed for each variable $x_{k}$. A large value of $\mu$ means that the parameter $x_{k}$ has an important overall effect on the output, while a large value of $\sigma$ is associated with non-linear effects or with strong interactions with other parameters [26]. The significant parameters can be analyzed using the $\mu-\sigma$ plane.

Results revealed that these model outputs were particularly sensitive to variations of parameters $C_{L}, C_{C W}, K_{1}$, and $K_{3}$ (figure 2). These parameters 
Table 1 Model parameter values evaluated from previous studies

\begin{tabular}{|c|c|c|c|}
\hline Parameters & Value & Context & References \\
\hline $\begin{array}{l}\mathrm{K} 1\left(\mathrm{~cm} \mathrm{H}_{2} \text { O.s. } L^{-1}\right) \\
\mathrm{K} 2\left(\mathrm{~cm} \mathrm{H}_{2} \text { O.s. } L-1\right) \\
\mathrm{Iu}\left(\mathrm{cm} H_{2} 0-1 . s^{2} \cdot L^{-1}\right)\end{array}$ & $\begin{array}{l}42 \\
465 \\
0.33\end{array}$ & Assessed on artificial newborn trachea & {$[15]$} \\
\hline $\operatorname{Vcmax}(\mathrm{L})$ & 0.008 & Estimation of total dead space & {$[5]$} \\
\hline $\mathrm{A}\left(\mathrm{cm} \mathrm{H}_{2} \mathrm{O}^{-1}\right)$ & 0.35 & Extrapolated from adult value & {$[3]$} \\
\hline $\mathrm{B}\left(\mathrm{cm} \mathrm{H}_{2} \mathrm{O}^{-1}\right)$ & 0.2 & Extrapolated from adult value & {$[3]$} \\
\hline $\mathrm{Rp}\left(\mathrm{cm} H_{2}\right.$ O.s. $L^{-1}$ & 0.02 & Extrapolated from adult value & {$[2]$} \\
\hline Rve $\left(\mathrm{cm} H_{2}\right.$ O.s. $\left.L^{-1}\right)$ & 1 & Extrapolated from adult value & {$[2]$} \\
\hline Cve (L.cm $\left.H_{2} \mathrm{O}-1 . \mathrm{kg}^{-1}\right)$ & 0.5 & Extrapolated from adult value & {$[2]$} \\
\hline
\end{tabular}

were thus identified first in order to have simulations as close as possible to the experimental data and to obtain a set of parameters specific to each newborn lamb. Moreover, it was necessary to identify the parameters characterizing respiratory muscle activity since, to our knowledge, no previous study has presented such analytical description. The set of specific parameters $\mathrm{P}$ that needed to be identified were thus defined as $\mathrm{P}=\left[C_{L}, C_{C W}, K_{1}, K_{3}, P_{\max }, \alpha\right.$, $\left.\beta, T_{I}\right]$.

\subsubsection{Identification algorithm}

An identification algorithm was used to estimate the optimal set of parameters $\mathrm{P}^{*}$ that minimizes the error function $(\epsilon)$ defined as the sum of two error functions $\epsilon=\epsilon_{S V}+\epsilon_{P P}$, where $\epsilon_{S V}$ and $\epsilon_{P P}$ are respectively the error functions computed during spontaneous ventilation and during the application of a positive pressure. In order to obtain the same order of magnitude for $\epsilon_{S V}$ and $\epsilon_{P P}$, signals were detrended and scaled. The error function regarding spontaneous ventilation can be determined as the sum of absolute values of the differences between each variable:

$$
\epsilon_{S V}=\frac{1}{N_{S V}} \sum_{t_{r}=t_{r 0}}^{t_{R E S P}}\left(\left|\left(\hat{\dot{P}}_{S V}^{s i m}\left(t_{r}\right)-\hat{\dot{P}}_{S V}^{\exp }\left(t_{r}\right)\right)\right|+\left|\left(\hat{\dot{V}}_{S V}^{\text {sim }}\left(t_{r}\right)-\hat{\dot{V}}_{S V}^{\text {exp }}\left(t_{r}\right)\right)\right|\right)
$$

where $t_{r}$ corresponds to the time elapsed since the onset of the current respiratory cycle, $t_{r 0}, t_{R E S P}$ are respectively the beginning and the end of the respiratory cycle and $N_{S V}$ is the number of samples of respiratory signals. The variables $\hat{\dot{P}}_{S V}^{\text {sim }}, \hat{\dot{P}}_{S V}^{\text {exp }}, \hat{\dot{V}}_{S V}^{\text {sim }}$ and $\hat{\dot{V}}_{S V}^{\text {exp }}$ correspond to the experimental and simulated derivative of esophageal pressure and flow during spontaneous ventilation, which have been detrended and scaled.

During the application of a positive pressure, the lambs are intubated. Thus, it is not necessary to identify the upper airway resistance $\left(K_{1}\right)$ since its 
value can be set to the tube resistance $R_{t}$ using Poiseuille's law $R_{t}=\frac{8 \cdot l \cdot \mu}{\pi \cdot r^{4}}$ where $\mu$ is the air viscosity, $l$ is the tube length equal to $17.8 \mathrm{~cm}$ and $r$ is the tube radius equal to $2.25 \mathrm{~mm}$. Hence, tube resistance is equal to $3.3 \mathrm{~cm} \mathrm{H}_{2}$ O.s. $L^{-1}$. In order to identify the other parameters, an additional error function is computed for the signals acquired during the application of a positive pressure:

$$
\epsilon_{P P}=\frac{1}{N_{P P}} \sum_{t_{v}=t_{v 0}}^{t_{v 0}+30 s}\left(\left|\left(\hat{\dot{P}}_{P P}^{s i m}\left(t_{v}\right)-\hat{\dot{P}}_{P P}^{\exp }\left(t_{v}\right)\right)\right|\right)
$$

where $t_{v}$ is the time elapsed and $t_{v 0}$ is the beginning of a 30 second-period, which includes the 15 seconds of positive pressure application, and $N_{P P}$ is the number of samples. The signals $\hat{\dot{P}}_{P P}^{\operatorname{sim}}$ and $\hat{\dot{P}}_{P P}^{\exp }$ stand respectively for the simulated and experimental detrended and scaled derivative of esophageal pressure during the application of positive pressure.

The error function $\epsilon$ had to be minimized in order to determine the optimal set of parameters $\mathrm{P}^{*}$. To solve this problem, we elected to use evolutionary algorithms, which are particularly adapted to complex nonlinear problems characterized by a poorly-known state-space. Evolutionary algorithms are stochastic search methods inspired from the natural selection process. In these algorithms, the set of parameters $\mathrm{P}$ (or chromosomes) characterizes each "individual" of a "population", which will evolve to approach the optimum [25] .

First, a set of random chromosomes was used to create the initial population. The parameters corresponding to each individual was generated using a uniform distribution bounded by feasibility intervals: [5 70] for $C_{L}$ $\left(\mathrm{mL} . \mathrm{cm} H_{2} \mathrm{O}^{-1}\right),[570]$ for $\mathrm{C}_{\mathrm{CW}}\left(\mathrm{mL} . \mathrm{cm} H_{2} \mathrm{O}^{-1}\right),[120]$ for $K_{1}\left(\mathrm{~cm} \mathrm{H}_{2} 0 . \mathrm{s}^{-1} . L^{-1}\right)$, [1 50] for $K_{3}\left(\mathrm{cmH}_{2} 0 . s^{-1} \cdot L^{-1}\right)$, [1 15] for $P_{\max }\left(\mathrm{cm} H_{2} 0\right)$, [0.1 30] for $\alpha$ (n.u.), [0.1 30] for $\beta$ (n.u.) and [0.1 2] for $T_{I}$ (s). These intervals were defined to approximate published values for mechanical parameters $\left(C_{L}, C_{C W}, K_{1}, K_{3}\right)$ and to be large enough for the parameters of the analytical function describing respiratory muscles $\left(P_{\max }, \alpha, \beta, T_{I}\right)[8][15][38]$.

Once the initial population was created, an iterative process was performed. The error function $\epsilon$, described previously, was computed at each iteration in order to evaluate the performance of each individual. Solutions with low error values were recombined with other solutions (cross-over) and small, random modifications of a chromosome were also introduced (mutation). In this process, the probability of selecting a given individual depends on the value of its error function, divided by the sum of all the error values of the population. A uniform crossover was used to create two new individuals (offspring) from two existing individuals (parents), by randomly copying each "allele" (i.e. parameter value) from one parent or the other, depending on a uniform random variable. A "Gaussian mutation" was chosen to create a new individual by randomly changing the value of one allele (selected randomly), based on a Gaussian distribution around the current value. The mutation operator helps the process to explore the entire search space and prevent from convergence to a local minimum. 

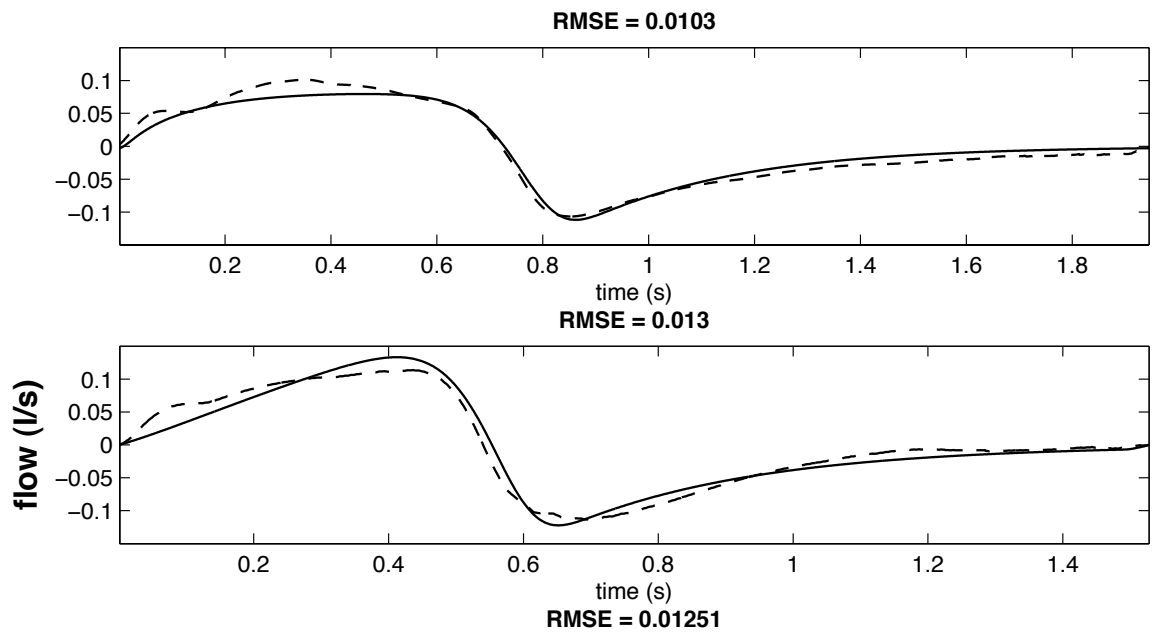

RMSE $=0.01251$

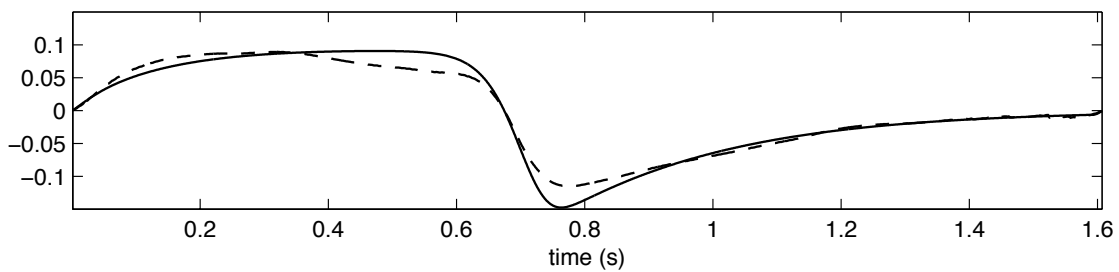

Fig. 3 Comparison of model flow simulations (continuous lines) with experimental nasal flow data (dotted lines) during spontaneous, stationary respiration in three lambs. The airflow signal was selected from the mean respiratory cycle in each lamb; the signal is positive during inspiration and negative during expiration. Deviations are given as RMSE in each lamb.

\section{Results}

Figures 3, 4 and 5 show the comparison and the Root Mean Square Error (RMSE) between simulated and experimental data acquired from the three lambs.

The average RMSE value between simulated and experimental nasal flow for one respiratory cycle in stationary respiratory conditions is equal to 0.0119 (Figure 3). The difference between simulated and experimental flow, in the regions where flow derivative changes much, can be explained by the parameter Iu, which has not been identified in the present study. Moreover, model simulations correctly predicted esophageal pressure variations and reproduced the experimental data in the three lambs because the average RMSE, between simulated and experimental $\mathrm{dPes} / \mathrm{dt}$ for one respiratory cycle, is equal to 0.0052 .

Finally, the simulated and experimental dPes/dt during CPP15 maneuver in three lambs are shown in Figure 5. Even if these signals are noisy, notably due to the derivation, the simulations reproduced the experimental data waveforms with an average value of RMSE equal to 0.0094. Parameters of res- 


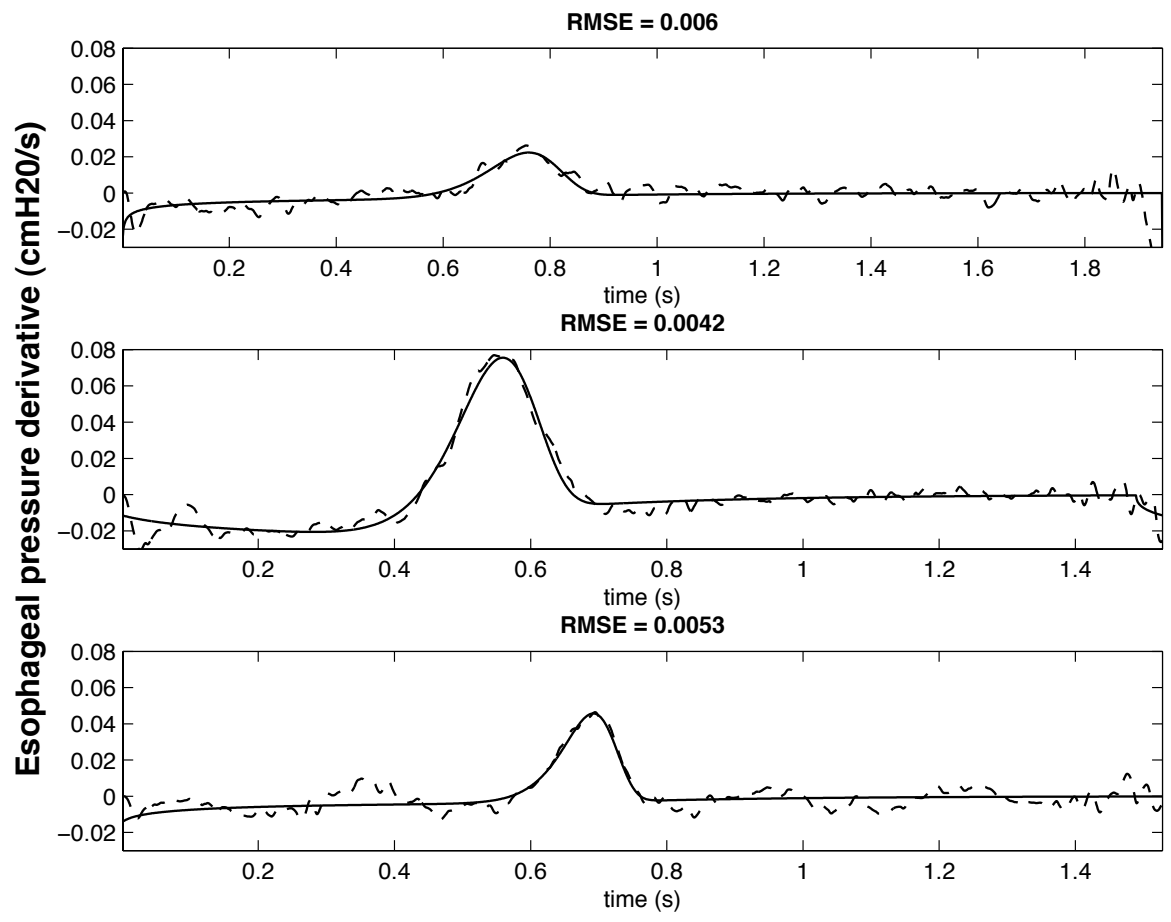

Fig. 4 Comparison of $\mathrm{dPes} / \mathrm{dt}$ model simulations (continuous lines) with experimental $\mathrm{dPes} / \mathrm{dt}$ data (dotted lines) during spontaneous respiration in three lambs. The esophageal pressure signal was selected from the mean respiratory cycle in each lamb. As esophageal pressure decreases during inspiration, the derivative is negative; the converse is seen during expiration. Deviations are given as RMSE in each lamb.

piratory mechanics identified with our neonatal model are presented in table 2 .

\section{Discussion}

\subsection{Model limitations}

The proposed lumped-parameter model presents an inherent low spatial resolution: in particular, all alveoli are represented by a single compartment. However, the proposed model is sufficiently detailed to reproduce the observed respiratory responses to the application of various levels of positive pressure, while presenting low computational costs. Moreover, this low spatial resolution is also justified by the well-known mechanical homogeneity of newborn lungs.

Chest wall properties were also assumed to be linear, although inward rib cage movement frequently occurs during inspiration in the newborn. Such rib cage distortion is mainly observed: i) during Rapid Eye Movement sleep (REM), when abolition of intercostal muscle tonic activity decreases rib cage 


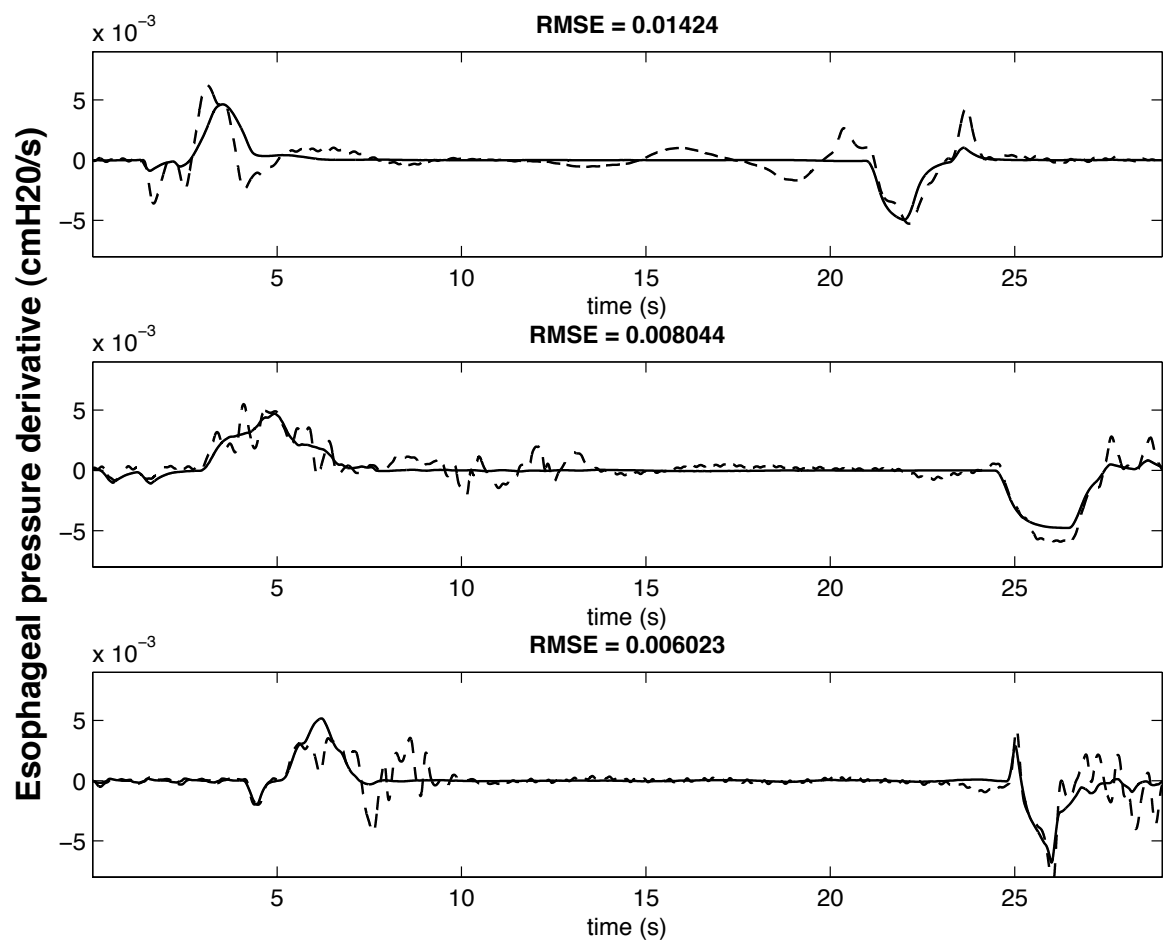

Fig. 5 Comparison of dPes/dt model simulations (continuous lines) with dPes/dt experimental data (dotted lines) during a CPP15 maneuver. The first positive peak corresponds to a Pes increase at the beginning of the maneuver whereas the negative peak stands for the Pes decrease at the end of the maneuver. Deviations are given as RMSE in each lamb. Total duration of the maneuver was 30 seconds.

stiffness, or ii) during respiratory distress, when increased inspiratory efforts induce highly negative intrathoracic pressures. In the presence of rib cage distortion, part of the greater inspiratory work developed in an attempt to preserve minute ventilation and prevent respiratory insufficiency does not translate directly into lung ventilation. Such dissipation of energy can lead to respiratory muscle fatigue, which is especially frequent at that age [13]. The analytic representation of respiratory muscle activity (Pmus) that we propose paves the way for the application of the model to conditions associated with such respiratory muscle fatigue. However, this would need to integrate the consequences of rib cage distortion on respiratory system mechanics and the accompanying dissipation of respiratory muscle energy into the model. Moreover, the individual contribution of each type of respiratory muscle involved in the generation of the total Pmus is not represented. Instead, the proposed analytic function has been designed to fit the different Pmus morphologies generated by the complex and heterogeneous activation of all respiratory muscles contributing to a given respiratory cycle (including eventually the abdominal muscles) and the abdominal pressure. Thus, this "black-box" approach has the advantage of 
Table 2 Parameters of respiratory mechanics identified with the present neonatal model.

\begin{tabular}{llll}
\hline & lamb 1 (3kg) & lamb 2 (3.6kg) & lamb 3 (4.5kg) \\
\hline Parameters of Respiratory Mechanics & & & \\
\hline$C_{L}\left(\mathrm{~L} . \mathrm{cm} \mathrm{H}_{2} \mathrm{O}^{-1} \cdot \mathrm{kg}^{-1}\right)$ & 0.00317 & 0.00441 & 0.00361 \\
$C_{C W}\left(\mathrm{~L} . \mathrm{cm} \mathrm{H}_{2} \mathrm{O}^{-1} \cdot \mathrm{kg}^{-1}\right)$ & 0.005 & 0.01242 & 0.01483 \\
$K_{1}\left(\mathrm{~cm} \mathrm{H}_{2} \mathrm{O} . \mathrm{s} . \mathrm{L}^{-1} \cdot \mathrm{kg}^{-1}\right)$ & 0.82 & 4.21 & 1.94 \\
$K_{3}\left(\mathrm{~cm} \mathrm{H}_{2} \mathrm{O} \cdot \mathrm{s} . \mathrm{L}^{-1} \cdot \mathrm{kg}^{-1}\right)$ & 15.33 & 2.02 & 3.04 \\
\hline Pmus Parameters & & & \\
\hline Pmax $\left(\mathrm{cm} \mathrm{H}_{2} \mathrm{O}\right)$ & 7.11 & 2.09 & 2.06 \\
$\alpha$ & 9.97 & 22.04 & 12.45 \\
$\beta$ & 1.38 & 0.68 & 0.70 \\
$T_{I}(\mathrm{~s})$ & 0.59 & 0.70 & 0.76 \\
\hline
\end{tabular}

fitting correctly the observed signals, but is limited in the interpretation of the relative contribution of each kind of muscle. Although this level of representation is adapted to the goal of this paper, the simulation of certain pathologies such as bowel distention or hepatomegaly would induce changes in the abdominal pressure which could not be represented in the current version of the model.

Of note, our model may not apply to newborn lambs in the first $48 \mathrm{~h}$ after birth, where respiratory mechanics undergo very rapid and dramatic changes due to lung aeration and constitution of the functional residual capacity, increase in pulmonary blood flow and closure of the ductus arteriosus. Such conditions include premature birth, especially due to incomplete alveolarization and deficient surfactant [30] [32], respiratory distress of the newborn and/or surfactant treatment [32].

\subsection{Dynamics of breathing in the newborn}

Results from the present study offer a model of neonatal respiratory mechanics, whose parameters have been specifically identified from experimental data obtained from newborn lambs during dynamic conditions. The generation of realistic waveforms is notably due to the analytical law describing respiratory muscle activity throughout the breathing cycle. Since the upper airway resistance is fixed during the CPP15 maneuver due to the need for endotracheal intubation, the discussion will mainly focus on the analysis of compliance values.

Several techniques can be used to estimate the static or dynamic values of respiratory mechanics based on measurements of flow, volume and esophageal pressure. Lung compliance values identified with our model are slightly higher than those reported in previous studies (Table 3). Differences may be explained by the diversity of experimental protocols (nasal mask vs. endotracheal tube, anesthesia vs. no sedation, postnatal age,) and natural inter-individual variability. Chest wall compliance $\left(C_{C W}\right)$ values identified in our model are sim- 
Table 3 Comparison of lung compliance $\left(C_{L}, \mathrm{~mL} . \mathrm{cm} \mathrm{H}_{2} \mathrm{O}^{-1} \cdot \mathrm{kg}^{-1}\right)$ and chest wall compliance $\left(C_{C W}, \mathrm{~mL} . \mathrm{cm} \mathrm{H}_{2} \mathrm{O}^{-1} . \mathrm{kg}^{-1}\right)$ values identified with the present model and those from previous studies in newborn lambs.

\begin{tabular}{lll}
\hline Lung Compliance $C_{L}$ & Chest wall Compliance $C_{C W}$ & Reference \\
\hline$C_{L}=3.7(+/-0.6)$ & $C_{C W}=10.7(+/-5.1)$ & Present model \\
$C_{L}=2.57(+/-0.58)$ & $C_{C W}=11.39(+/ 1.70)$ & {$[8]$} \\
$C_{L}=2.21(+/-0.25)$ & $C_{C W}=11.3(+/ 0.97)$ & {$[8]$} \\
$C_{L}=2.91(+/-0.19)$ & $C_{C W}=6.4(+/-0.71)$ & {$[18]$} \\
$C_{L}=1.55(+/-0.04)$ & $C_{C W}=7.37(+/-1.31)$ & {$[7]$} \\
\hline
\end{tabular}

ilar [8][9] or higher [7] than those reported previously (Table 3). Again, the variability between previously reported values can be explained by the different experimental protocols and measurement methods. In particular, $C_{C W}$ is highly dependent on both tonic and phasic chest wall muscle activity. Overall, the present study illustrates that, under the various breathing conditions used in the experimental protocol, the model-predicted compliances $C_{L}$ and $C_{C W}$ are comparable to those obtained with other measurement methods (see table 3). Moreover, the model-predicted parameters show that lung compliance is lower than chest wall compliance. Such characteristics are known to be specific to the neonatal period [11], the elevated chest wall compliance being explained by the incomplete ossification of the rib cage.

One consequence of $C_{C W}$ being higher than $C_{L}$ is the increased occurrence of chest wall distortion in the newborn. In order to compensate for the latter, respiratory muscles must then develop greater force to stabilize the chest wall. Though important at that age, chest wall distortion has not been included in our model. One advantage of the present model is to provide compliance values adapted for various breathing conditions. Indeed, the model is able to reproduce experimental respiratory data both during spontaneous ventilation and CPP15 maneuvers. Moreover, whereas other methods [8] [9] take into consideration only a few particular elements of respiratory signals, the proposed method requires the entire signal waveform to determine compliance values.

\section{Conclusion}

In this paper, a lumped-parameters model, including lung and chest wall compliances, has been presented. The model structure is based on other studies but the main originality of this work is to propose a sensitivity analysis and a parameter identification in order to adapt the model to the neonatal period. The identified mechanical parameters are coherent with neonatal physiological knowledge. The other specificity of this work is to simulate realistic signal profiles for a wide range of breathing conditions. This is mainly due to the analytic law, describing the respiratory muscle dynamic, proposed and coupled with the mechanical model. Current work is directed to the integration of i) the increased lung tissue resistance, rib cage distortion and respiratory muscle fatigue, which are characteristic of the newborn physiology, and ii) 
the coupling of the proposed model with a cardiovascular model allowing to study neonatal cardiopulmonary interactions in health and disease. The ability of the respiratory model to reproduce realistic signal profiles will allow a beat-to-beat signals analysis of pulsatile cardiovascular variables (arterial pressure,...), that are directly influenced by respiration, especially during the application of mechanical ventilation on newborn infants.

Acknowledgements The authors acknowledge Jean-Philippe Gagne for expert technical assistance.

\section{References}

1. Asher M., Coates A., Collinge J., and Milic-Emili J. Measurement of pleural pressure in neonates, J. Appl. Physiol., 52:491-494 (1982)

2. Athanasiades A., Ghorbel F., Clark J. W. J., Niranjan S. C., Olansen J., Zwischenberger J. B., and Bidani A., Energy analysis of a nonlinear model of the normal human lung, J. Biol. Syst., 8:115-139 (2000)

3. Avanzolini G., Barbini P., Bernardi F., Cevenini G., and Gnudi G., Role of the mechanical properties of tracheobronchial airways in determining the respiratory resistance time course, Ann. Biomed. Eng., 29:575-586 (2001)

4. Calabrese P., Baconnier P., Laouani A., Fontecave-Jallon J., Guméry P. Y., Eberhard A., and Benchetrit G., A simple dynamic model of respiratory pump, Acta Biotheor., 58:265-275 (2010)

5. Costantino M. L., Bagnoli P., Dini G., Fiore G. B., Soncini M., Corno C., Acocella F., and Colombi R., A numerical and experimental study of compliance and collapsibility of preterm lamb tracheae, J. Biomech., 37:1837-1847 (2004)

6. Crooke P. S., Marini J. J., and Hotchkiss J. R., Modeling Recruitment Maneuvers with a Variable Compliance Model for Pressure Controlled Ventilation, Comput. Math. Methods. Med., 4:197-207 (2002)

7. Davey M. G., Johns D. P., and Harding R, Postnatal development of respiratory function in lambs studied serially between birth and 8 weeks, Respiration Physiology, 113:83-93 (1998)

8. Davis G. M., Coates A. L., Dalle D., and Bureau M. A., Measurement of pulmonary mechanics in the newborn lamb: a comparison of three techniques, Journal of Applied Physiology, 64:972-981(1988)

9. Davis G. M., Coates A. L., Papageorgiou A., and Bureau M. A., Direct measurement of static chest wall compliance in animal and human neonates, Journal of Applied Physiology, 65:1093-1098 (1988)

10. Fontecave-Jallon J., Abdulhay E., Calabrese P., Baconnier P., and Guméry P. Y., A model for mechanical interactions between heart and lungs, Philos. Transact. A Math. Phys. Eng. Sci., 367:4741-4757 (2009)

11. Frappell P. B. and MacFarlane P. M., Development of mechanics and pulmonary reflexes, Respir. Physiol. Neurobiol., 149:143-154 (2005)

12. Gappa M., Jackson E., Pilgrim L., Costeloe K., and Stocks J., A new microtransducer catheter for measuring esophageal pressure in infants, Pediatr. Pulmonol., 22:117124 (1996)

13. Gaultier C. and Praud J. P., Respiratory pathology during sleep in children, Rev Mal Respir, 7:475-481 (1990)

14. Golden J., Mathematical Modelling of pulmonary airway dynamics, Rice University., 1972 .

15. Guttmann J., Kessler V., Mols G., Hentschel R., Haberthur C., and Geiger K., Continuous calculation of intratracheal pressure in the presence of pediatric endotracheal tubes, Crit. Care Med., 28:1018-1026 (2000) 
16. Hernández AI, Le Rolle V., Defontaine A., and Carrault G., A multiformalism and multiresolution modelling environment: application to the cardiovascular system and its regulation, Philosophical Transactions Mathematical Physical and Engineering Sciences, 367:4923-4940 (2009)

17. Hernández AI, Le Rolle V, Ojeda D, Baconnier P, Fontecave-Jallon J, Guillaud F, Grosse T, Moss RG, Hannaert P, Thomas SR. Integration of detailed modules in a core model of body fluid homeostasis and blood pressure regulation, Prog Biophys Mol Biol. 107(1):169-82 (2011)

18. Jakubowska A. E., Billings K., Johns D. P., Hooper S. B., and Harding R. Respiratory function in lambs after prolonged oligohydramnios during late gestation, Pediatr. Res.,34:611-617 (1993)

19. Khirani S., Biot L., Eberhard A., and Baconnier P., Positive end expiratory pressure and expiratory flow limitation: a model study, Acta Biotheor, 49:277-290 (2001)

20. Le Rolle V., Hernández A. I., Carrault G., Samson N., and Praud J. P., A model of ventilation used to interpret newborn lamb respiratory signals, IEEE EMBC, Vancouver 2008

21. Liu C. H., Niranjan S. C., Clark J. W. J., San K. Y., Zwischenberger J. B., and Bidani A., Airway mechanics, gas exchange, and blood flow in a nonlinear model of the normal human lung, J. Appl. Physiol., 84:1447-1469 (1998)

22. Lu K., Clark J. W. J., Ghorbel F. H., Ware D. L., and Bidani A., A human cardiopulmonary system model applied to the analysis of the Valsalva maneuver, Am. J. Physiol. Heart Circ. Physiol., 281:2661-2679 (2001)

23. Manilal-Reddy P. I. and Al-Jumaily A. M., Understanding the use of continuous oscillating positive airway pressure (bubble CPAP) to treat neonatal respiratory disease: An engineering approach, J. Med. Eng. Tech., 33:214-222 (2009)

24. Mead J., Contribution of compliance of airways to frequency-dependent behavior of lungs, J. Appl. Physiol., 26:670673 (1969)

25. Michalewicz Z., Genetic Algorithms + Data Structures = Evolution Programs NewYork, (1994)

26. Morris M. D., Factorial sampling plans for preliminary computational experiments, Technometrics, 33:161-174 (1991)

27. Mortola J., Dynamics of breathing in newborn mammals, Physiol Rev., 67:187-243 (1987)

28. Mount L., The ventilation flow-resistance and compliance of rat lungs, J. Physiol., 127:157-167 (1955)

29. Otis B., Mckerrow C. B., Bartlett R. A., Mead J., Selver-Stone M. B., McIlroy N. J., and Radford E. P. J., Mechanical factors in distribution of pulmonary ventilation, J. Appl. Physiol., 8:427-443 (1956)

30. Pillow J. J., Hall G. L., Karen E. W., Jobe A. H., Hantos Z., and Sly P. D., Effects of Gestation and Antenatal Steroid on Airway and Tissue Mechanics in Newborn Lambs, Am. J. Respir. Crit. Care Med., 163:1158-1163 (2001)

31. Pillow J. J., Sly P. D., Hantos Z., and Bates J. H. T., Dependence of Intrapulmonary Pressure Amplitudes on Respiratory Mechanics during High-Frequency Oscillatory Ventilation in Preterm Lambs, Pediatr. Res., 52:538-544 (2002)

32. Pillow J. J., Stocks J., Sly P. D., and Hantos Z., Partitioning of Airway and Parenchymal Mechanics in Unsedated Newborn Infants, Pediatr Res, 58:12101215 (2005)

33. Quatember B., Human Respiratory System: Simulation of Breathing Mechanics and Gas Mixing Processes Based on a Non-linear Mathematical Model, International Conference on Health Sciences Simulation (2003).

34. Riddle W. and Younes M., A model for the relation between respiratory neural and mechanical outputs. II. Methods, J Appl Physiol., 51:979-989 (1981)

35. Robert R., Modélisation numérique et stratégies de commande du débit expiratoire pour éviter le collapsus des voies respiratoires en ventilation liquidienne totale, University of Sherbrooke, 2007.

36. Rozanek M. R. and Roubik K., Influence of The Changes in Pulmonary Mechanics upon the Suitability of Artificial Lung Ventilation Strategy, Biomedical Engineering, 417-132 (2004) 
37. Schmidt M., Foitzik B., Hochmuth O., and Schmalisch G., Computer simulation of the measured respiratory impedance in newborn infants and the effect of the measurement equipment, Med. Eng. Phys., 20:220-228 (1998)

38. Sly P. D., Brown K. A., Bates J. H. T., Spier S., and Milic-Emili J., Noninvasive determination of respiratory mechanics during mechanical ventilation of neonates: a review of current and future techniques, Pediatr. Pulmonol., 4:39-47, (1988)

39. Thibault S., Calabrese P., Benchetrit G. A., and Baconnier P., Effects of resistive loading on breathing variability : non linear analysis and modelling approaches., Adv. Exp. Med. Biol., 551:293-298 (2004)

40. Thibault S., Heyer L., Benchetrit G. A., and Baconnier P., Ventilatory Support: A Dynamical Systems Approach, Acta Biotheor, 50:269-279 (2002)

41. Tse Ve Koon K., Thebault C., Le Rolle V., Donal E., and Hernández A., Atrioventricular Delay Optimization in Cardiac Resynchronization Therapy Assessed by a Computer Model, Computers in Cardiology Belfast, 333?336, Ireland 2010

42. Verbraak A. F., Bogaard J. M., Beneken J. E., Hoorn E., and Versprille A., Serial lung model for simulation and parameter estimation in body plethysmography, A. Med. Biol. Eng. Comput., 29:309-317 (1991)

43. Winkler T., Krause A., and Kaiser S., Simulation of mechanical respiration using a multicompartment model for ventilation mechanics and gas exchange, Int. J. Clin. Monit. Comput. 12:231-239, (1995)

44. Younes M. and Riddle W., A model for the relation between respiratory neural and mechanical outputs. I. Theory., J Appl Physiol., 51:963-978 (1981) 